\title{
Tendencias en ciencias de la computación
}

\section{Computer science trends}

\author{
Blandón, J. C. \\ Recibido: noviembre 15 de 2019 - Aceptado: marzo 20 de 2020
}

\begin{abstract}
Resumen- Un estudio de tendencias consiste en revisar y analizar gran cantidad de información de una disciplina, desde diferentes fuentes de información. En este artículo se muestran los principales temas tendencia en ciencias de la computación a nivel mundial, teniendo en cuenta los avances de la industria 4.0. La literatura existente plantea un gran interés por la aplicación práctica de temas teóricos de la academia en el sector productivo y gubernamental. Se hace necesario presentar los temas de tendencia actuales en distintos sectores y contextos. De esta manera, se presenta un estudio desde la perspectiva de los gobiernos, la empresa y la academia, el cual evidencia los temas pertinentes en ciencias de la computación. La intención es que sirva como insumo importante para una articulación entre el sector académico, gubernamental y productivo para una posterior contribución en el desarrollo de los países.
\end{abstract}

Palabras clave- Brecha digital, computación, desarrollo económico, industria 4.0, innovación, retos.

Abstract - A trend study consists to review and analyze a great amount of information that refers to a discipline, it is based on many sources of information. This paper shows the main trend topics in computer science around the world, this base on the advances in industry 4.0. The background shows a great interest in the practical application of theoretical topics studied in the academy inside the productive and governmental sectors. Then, it is relevant to present the current trend issues in many sectors and contexts. In this work, a study is presented from the perspective of governments, business, and academia, which evidences the relevant topics for computer sciences. This article pretends to contribute to the articulation between the academic, governmental, and productive sectors as a contribution to the development of the countries.

${ }^{1}$ Producto derivado del studio denominado "Estudios de Tendencias Universidad Católica de Pereira" realizado en el grupo de Investigación Entre Ciencia e Ingeniería de la Facultad de Ciencias Básicas e Ingeniería.

J. C. Blandón. Universidad Católica de Pereira, Pereira, Colombia. e-mail: juanc.blandon@ucp.edu.co

Como citar este artículo: Blandón, J. C. Tendencias en ciencias de la computación, Entre Ciencia e Ingeniería, vol. 14, no. 27, pp. 19-28, enerojunio 2020. DOI: https://doi.org/10.31908/19098367.1740.

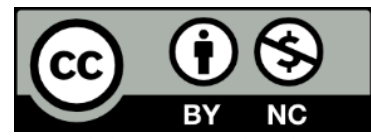

Attribution-NonCommercial 4.0 Intenational (CC By-NC 4.0)
Keywords - Digital divide, computing, economic development, industry 4.0, innovation, challenges.

\section{INTRODUCCIÓN}

Según Sachon [1], con la tecnología es posible conectar máquinas, productos e infraestructura, de tal forma que haya una colaboración dinámica y que las empresas sean más productivas. Por otro lado, Salimi et al. [2] realiza una crítica a los métodos para cuantificar la incertidumbre, los autores buscan aportar ideas acerca de cómo la tecnología puede aportar al sector productivo. Chang et al. [3] presenta algunas tendencias en tecnología y cómo ellas podrían impactar el sector económico de los países. Adicional a esto, en [4] se presentan algunos desafíos para el año 2030, ello con el propósito de reflexionar sobre las oportunidades que existen cuando se integra la ciencia y la tecnología con la educación. Por otra parte, el currículo para la tecnología de la información 2017 [5], presenta temas generales de tendencia para la construcción de un currículo que aporte al sector productivo.

Así, se puede decir que la tecnología es un factor determinante actualmente para la sociedad, y también para el sector productivo, que necesita los adelantos tecnológicos de la academia, pero que ellos sean aplicados de forma práctica en sus procesos productivos. En Colombia, la tecnología se relaciona con los programas de Ingeniería de Sistemas los cuales se desarrollan teniendo en cuenta áreas de conocimiento como ciencias de la computación, ingeniería de software y sistemas de información. También es necesario mencionar que a nivel mundial estos programas se conocen con el nombre de ciencias de la computación [6]. Teniendo en cuenta lo mencionado, el interés es revisar la disciplina a nivel mundial, como consecuencia de ello este documento se enfoca hacia ciencias de la computación.

En este trabajo se realiza un estudio de tendencias en el área, se busca identificar los principales temas importantes y de actualidad a los que apuestan los gobiernos, las empresas y la academia a nivel mundial para solucionar problemáticas reales y que a largo plazo contribuyen en el desarrollo de los países.

Con base en todo lo anterior, el presente artículo es un documento que pueden utilizar los diferentes sectores relacionados con la tecnología y en especial con las temáticas 
en ciencias de la computación, el objetivo es que puedan identificar hacía donde deben enfocar sus esfuerzos, para contribuir con el crecimiento económico de sus sectores y en general de sus países.

Por consiguiente, este artículo está organizado de la siguiente forma: en la Sección II se presenta el marco teórico. En la sección III se expone la metodología utilizada. En la sección IV se desarrolla el proceso de revisión. Luego se proporciona una síntesis de datos y análisis de resultados en la sección V. Finalmente, en la sección VI se presentan las conclusiones.

\section{TRABAJO RELACIONADO}

\section{A. Marco Teórico}

\section{1) Estudios de tendencias de una disciplina}

Los estudios de tendencias buscan llamar la atención de las personas que están relacionadas con muchos sectores. Un trabajo de este tipo, debe llevar inmerso una revisión y diagnóstico del estado del arte en las diferentes realidades mundiales desde el sector público y privado, y cómo la academia podría aportar para el crecimiento de la industria, en términos de mejorar la calidad de vida de las personas [6], [7].

\section{2) Currículo}

El currículo "es una construcción social e histórica que representa una idea de ser humano y sociedad, por esto es un espacio de conflicto. Su diseño es cultural y en ocasiones se limita a formar para el trabajo, pero también se plantea como una instancia de emancipación que revela la ideología subyacente porque reproduce las pautas de conducta del poder político y económico" [8]. En otras palabras "En el currículo escolar se plasman los fines de la educación que se pretenden materializar mediante la descripción de los contenidos, métodos, objetivos, conocimientos y destrezas" [8].

\section{3) Industria 4.0}

La cuarta revolución industrial, la cual, según [9], hace referencia a las tecnologías digitales y de automatización, las cuales son consecuencia del crecimiento importante de los teléfonos inteligentes, innovaciones tecnológicas que suceden rápidamente, y la gran cantidad de información que se encuentra en la red y que es tratada como Big Data. Todo ello está generando cambios muy grandes en el estilo de vida de las personas y, por consecuencia, en las sociedades. Mediante el uso de tecnologías emergentes, los gobiernos pueden reducir costos, al mismo tiempo que mejoran los servicios que ofrecen, debido a que serán más racionales, efectivos, colaborativos, transparentes y democráticos [10].

\section{4) Ciencias de la Computación}

$\mathrm{El}$ avance de la industria 4.0 se debe en gran medida al avance de diferentes dispositivos, que juegan un papel protagónico, debido al éxito de los sistemas de cómputo en el procesamiento de información [11]. La Universidad de Maryland define las ciencias de la computación como el estudio de los computadores y los sistemas computacionales. Con base en lo anterior, se puede decir que los ingenieros en computación se ocupan principalmente de software y sistemas de software, incluyendo su teoría, diseño, desarrollo y aplicación. También se menciona que entre las principales áreas de estudio se encuentran: inteligencia artificial, seguridad, sistemas de bases de datos, interacción humano computador, visión y gráficos, análisis numérico, lenguajes de programación, ingeniería de software, bioinformática y teoría de la computación.

En este mismo tema, otra definición es la de Correa-Henao et al.[6], quien afirma que "las ciencias de la computación buscan a través de la combinación de varias disciplinas, crear y estudiar sistemas complejos de información tecnológica que se materializan en programas de computación no tangibles conocidos como software".

\section{Metodología}

En este estudio se muestran los temas relevantes en la actualidad para la disciplina de ciencias de la computación a nivel mundial. Se tratarán dos aspectos fundamentales: i) estado del arte y ii) desafíos actuales. Para el estado del arte se realiza una búsqueda bibliográfica en los sectores gubernamental, empresarial y académico. Se utiliza una de las clasificaciones representativas de cada sector, ello con el fin de tomar información de las instituciones que se destacan. Para los desafíos actuales, se extrae información desde distintas fuentes con el fin de contribuir en el desarrollo de la disciplina de acuerdo a los retos que se presentan en la actualidad.

Se propone, entonces, una metodología basada en tres fases, a saber.

- Exploración: se realiza la revisión documental.

- Síntesis: se tabulan los temas actuales y que son relevantes para la disciplina.

- Conclusiones: se presentan los aspectos relevantes de la revisión realizada, para que puedan ser tenidos en cuenta.

\section{PROCESO DE REVISIÓN}

\section{A. Sector Gubernamental}

Para analizar y medir los factores que rigen la digitalización de un país se puede utilizar el índice de evolución digital [12]. Los países que encabezan esa clasificación en el año 2017, son en su orden: Noruega, Suecia, Suiza, Dinamarca, Finlandia, Singapur, Corea del Sur, Hong Kong, Estados Unidos, Australia, Canadá, Holanda, Nueva Zelanda y Japón, como los primeros 15 del Ranking conformado por 60 países.

Por otra parte, en el reporte global de Tecnologías de la Información (TI), documento producto del Foro Económico Mundial [13], se conforma una clasificación donde se mide la capacidad que tiene un estado para realizar avances importantes en tecnologías de la información y lograr con ello una mayor competitividad, como consecuencia de la medición se busca reducir la brecha tecnológica y con ello mejorar la calidad de vida de sus habitantes. Según el Ranking de 139 
países, los que lideran la competitividad tecnológica son: Singapur, Finlandia, Suecia, Noruega y Estados Unidos. A continuación, se realizará una breve descripción de lo que realizan algunos de esos países en tecnología.

Por ejemplo, en Singapur el Gobierno ha hecho una gran apuesta aproximadamente desde el año 2010, para adoptar tecnologías y permear con ella a todas las regiones del país. La telefonía móvil ha logrado una tasa de penetración del $148 \%$. También ha realizado una apuesta grande para mejorar su agencia de investigación y que sus temas estén enfocados en medicina, alimentación de su población y avances clínicos utilizando las TI.

Finlandia logró que empresarios y gobernantes trabajaran juntos, con ello decidieron liderar procesos de nuevos materiales, física de baja temperatura, investigaciones del cerebro y genética. Han logrado realizar importantes avances en los temas que tienen que ver con banda ancha [13]. Por otra parte, en Suecia la Ingeniería goza de gran importancia, por lo tanto, se destinan muchos recursos del estado para fortalecer la industria TI. Un ejemplo es que cuentan con muchas patentes, más de 33-mil y la mayoría están relacionadas con áreas de TI. Aproximadamente el $98 \%$ de sus habitantes cuenta con una conexión a Internet. Existe un acuerdo entre el sector público y privado, consiste en invertir el $4 \%$ del Producto Interno Bruto (PIB) a la investigación en TI.

Por su parte, Noruega tiene una economía muy fuerte que está basada en la mejora de la infraestructura TI, los ingresos provenientes de esa actividad hacen que el PIB de esta nación haya crecido un $100 \%$ en los últimos años. De cada 100 habitantes 96 cuentan con servicio de Internet, con una velocidad promedio de 21.3 Megabytes por segundo. Muchas empresas noruegas aprovechan el alto grado académico de sus habitantes y utilizan la tecnología para hacer que sus negocios sean más productivos.

Desde hace muchos años, Estados Unidos alberga la mayor cantidad de empresas de tecnología del mundo, es el caso de Silicon Valley, que agrupa varias empresas de base tecnológica como Google, Amazon, Uber, entre otras. Son líderes en las clasificaciones relacionadas con el uso de tecnologías como computadores, teléfonos móviles y telecomunicaciones. También se preocupan desde hace unos años en la capacitación de la gente para Big Data, además las fuerzas militares siempre han estado a la vanguardia del uso de la tecnología, para utilizarla en el campo de batalla. Este país es líder en artículos de investigación científica en tecnología y se resalta como el país que tiene la conexión de banda ancha más económica con un costo de 16 dólares, es un bajo costo teniendo en cuenta que el promedio mundial es 52 dólares.

En el reporte global de Tecnologías de la Información [13], también se menciona que Inglaterra tiene 17 empresas de Big Data; Japón tiene la mayor cantidad de robots trabajando en la industria de los automóviles; Holanda tiene una red pública utilizada para Internet de las Cosas (IoT); Suiza tiene una gran superficie de centro de datos; Alemania se destaca por usar la tecnología para sus negocios y el impacto de las Tecnologías de la Información y la Comunicación (TIC) en el acceso a los servicios básicos; Francia es el líder en la prestación de servicios en línea a sus ciudadanos y uno de los mejores en términos de permitir su participación electrónica en el proceso de decisión del Gobierno. En el Ranking entre los países latinoamericanos Chile ocupa el puesto 38, Uruguay el 43, Panamá el 55, Colombia el 68, Brasil el 72, México el 76, Ecuador el 82, Argentina el 89, Perú el 90, Paraguay el 105, Venezuela el 108, Bolivia el 111. Una de las conclusiones del estudio, es que existe la firme necesidad de avanzar en TI en los países, esto con el fin de ser más competitivos y tratar de mejorar con ello la calidad de vida de los habitantes del planeta.

Con todo lo anterior, se puede mencionar que los gobiernos quieren mejorar los servicios que ofrecen y optimizar procesos mediante el uso de las tecnologías de información que tienen en las respectivas oficinas de los estados. Se quiere que los gobiernos cuenten con herramientas tecnológicas de punta, para lograrlo es necesario llevar a cabo proyectos ambiciosos en TI, para tratar de mejorar la relación existente entre proyectos, las personas y las entidades [14]. Los gobiernos en general, muestran interés por utilizar las TI para mejorar la calidad de vida de las personas lo cual es un paso importante hacia el desarrollo de los países.

\section{B. Sector empresarial.}

Según la revista Forbes 2018 [15], las personas más ricas del mundo son en su orden: Jeff Bezos, director ejecutivo de Amazon, con una fortuna estimada en 112 billones de dólares; en segundo lugar aparece Bill Gates, cofundador de Microsoft con una fortuna estimada de 90 billones de dólares; en quinto lugar Mark Zuckerberg, creador de Facebook, con una fortuna estimada en 71 billones de dólares; en séptimo lugar Carlos Slim, que tiene negocios en la industria de las comunicaciones y su fortuna se calcula en 67,1 billones de dólares; en el décimo lugar Larry Ellison, fundador de Oracle, con una fortuna de 58,5 billones de dólares; en el décimo segundo lugar aparece Larry Page, cofundador de Google, con una fortuna estimada en 48,8 billones de dólares; en el décimo tercer puesto aparece Sergey Brin, cofundador de Google, y con una fortuna estimada en 47,5 billones de dólares.

Por otra parte, la empresa independiente de consultoría Brand Finance, publica un Ranking anual [16] sobre las marcas más valiosas del mundo con su respectivo valor en el mercado. Los resultados de la clasificación en el año 2018 se presentan en la Tabla I.

TABLA I

RANKING DE MARCAS MÁS VALIOSAS DEL MUNDO[16]

\begin{tabular}{|c|c|c|}
\hline Ranking & Empresa & $\begin{array}{c}\text { Valor en el mercado (mil millones de } \\
\text { dólares) }\end{array}$ \\
\hline $\mathbf{1}$ & Amazon & 150,8 \\
\hline $\mathbf{2}$ & Apple & 146,3 \\
\hline $\mathbf{3}$ & Google & 120,9 \\
\hline $\mathbf{4}$ & Samsung & 92,3 \\
\hline $\mathbf{5}$ & Facebook & 89,6 \\
\hline $\mathbf{6}$ & AT\&T & 82,4 \\
\hline $\mathbf{7}$ & Microsoft & 81,1 \\
\hline $\mathbf{8}$ & Verizon & 62,8 \\
\hline $\mathbf{9}$ & Walmart & 61,5 \\
\hline $\mathbf{1 0}$ & ICBC & 59,2 \\
\hline
\end{tabular}

La empresa Amazon es una empresa dedicada al comercio electrónico (e-commerce), presta servicios de computación en la nube, también ha diversificado sus líneas de negocio y ha 
comprado otras empresas. También ha creado su asistente virtual llamado Alexa. Sus ganancias radican principalmente en que cobran un porcentaje en el precio de venta de los productos que comercializan en su sitito Web, también cobra a las empresas para que puedan anunciar sus productos [17]. Amazon Go es una de las tecnologías que se aplicó en las tiendas, la idea principal es que será un supermercado sin líneas de caja, es decir, los clientes pueden entrar utilizando una aplicación móvil como llave de acceso y ver su factura al salir sin los habituales cajeros. Para aplicar esta tecnología se utilizan conceptos como la visión de máquina que se encarga del reconocimiento facial, sensores y el aprendizaje profundo (Deep Learning) [18]. Este concepto apunta a lo que se llama hoy día ciudades inteligentes que busca orientar la vida hacia ambientes sostenibles, es decir, disminuir el consumo energético y emisión de gases [19]. Amazon Go, es un complejo sistema basado en su mayoría en la utilización de Inteligencia Artificial [20], también se aplican conceptos como Big Data y Analítica de Datos.

Por otro lado, aparece Apple, que es una empresa líder en tecnología y aunque sus productos finales son de tipo hardware, se tiene que trabajar muy fuerte en la creación de aplicaciones software, las cuales permitan potencializar esas creaciones. En la conferencia anual de desarrolladores Apple WWDC 2018, se presentaron las novedades de los productos que comercializa la compañía. Tim Cook presidente de Apple, lanzó una frase "Hoy día lo importante es el software", con ello quería presentar los nuevos sistemas operativos iOS 12 y MacOS Mojave que tienen el doble de velocidad y estarán centrados en realidad aumentada e inteligencia artificial. También se presentan algunas actualizaciones para las plataformas WatchOS, MacOS y TvOS, así como algunos atajos desarrollados para SIRI, el asistente personal inteligente de los dispositivos Apple. En general, la empresa hace grandes apuestas por temas como la inteligencia artificial, realidad aumentada, realidad virtual y edge computing [21].

Google es una compañía que ha incursionado en la creación de nuevas aplicaciones tales como maps, gmail, calendar, traslate, entre otras [22]. Para el año 2018 en la conferencia Google I/O, se presentaron varias novedades, entre ellas un prototipo de una función de Google Assistant llamada Duplex. También fue presentada una función de realidad aumentada, que permite usar los teléfonos Android, para jugar con otra persona en un dispositivo iOS de manera simultánea en un mismo espacio virtual. También se rediseñó la aplicación de News, la cual es más personalizada, ofrece más fuentes confiables de información y con ello trata de combatir las noticias falsas (fake news). Para el sistema operativo Android $\mathrm{P}$, se presentó una aplicación llamada Dashboard, que sirve para medir el tiempo de uso del dispositivo móvil, así ayudar a combatir la adicción al Smartphone. A rasgos generales, la compañía le apuesta fuertemente a la inteligencia artificial, realidad aumentada, vehículos autónomos mediante el uso de machine learning y realidad virtual [23].

Samsung en la conferencia CES realizada en 2018, presentó varios productos, por ejemplo, Bixby su asistente de voz, la cual fue presentada inicialmente en el teléfono Galaxy
S8 y la cual aparecerá en algunos electrodomésticos, también se mencionó SmartThings Cloud, que permitiría controlar dispositivos de internet de las cosas (IoT), desde una sola aplicación utilizando la red de Internet. La empresa muestra interés por las tecnologías disruptivas, a su vez presenta nuevos dispositivos como televisores, computadores, electrodomésticos, dispositivos de audio, entre otros. En general, la empresa quiere conectar los electrodomésticos a través del software SmartThings, que es la idea de Internet de las cosas, muchos de sus productos manejan inteligencia artificial, pero la empresa hace una gran apuesta por utilizar el término "inteligencia de las cosas", lo cual quiere decir la unión de los conceptos de internet de las cosas con inteligencia artificial [24], [25].

Facebook es una de las redes sociales más grande que existe en la actualidad. Una de las fortalezas de la empresa es la venta de publicidad y sobre todo la información privada que tiene guardada de las personas que tienen una cuenta de Facebook. La empresa constantemente crea aplicaciones para que la experiencia social de los usuarios sea mejor. En la conferencia F8 se presentaron algunas nuevas funciones. La primera es "Clear History", una herramienta que permite eliminar el historial de búsquedas y navegación, esta información era usada por los anunciantes y la plataforma para ofrecer productos y servicios. En la conferencia se hizo énfasis en la seguridad de la información y los cuidados que deben tener los usuarios. Entre otras novedades se presenta "Watch Party", que se puede utilizar para ver eventos televisivos con otros usuarios de la red, así como nuevas funciones para concertar citas. A grandes rasgos, se presentaron adelantos en realidad aumentada, realidad virtual, inteligencia artificial y algunas funciones restringidas en las que se usa Big Data [26], [27].

Microsoft para 2018 participa en la conferencia E3, la cual es la más famosa sobre videojuegos. Microsoft presentó varias creaciones, entre ellos: Gears 5, Cyberpunk 2077, Halo Infinite, Devil May Cry 5, con los cuales logró cautivar a muchas personas [28]. Microsoft Build, es una conferencia donde se reúnen cada año ingenieros de software y desarrolladores web de Microsoft, en 2018 giró en torno a la inteligencia artificial, servicios en la nube, su asistente de voz Cortana, Office y, por supuesto, Windows. Se presentó la herramienta Microsoft Layout, la cual permite crear modelos 3D con Realidad Aumentada para modelos de negocio. En cuanto a los conceptos de Intelligent Cloud e Intelligent Edge, la empresa abrió el código de Azure IoT Edge Runtime, ello con el fin de que los clientes puedan modificar, depurar y controlar sus aplicaciones, por ejemplo, permitir a drones y equipo industrial tomar acciones críticas sin conexión a la nube; también anunció una alianza estratégica con la empresa China de drones DJI, para lo cual construirá un kit de desarrollo para Windows 10, el cual permitirá conectar los drones con el sistema operativo; Otro avance, es el esfuerzo con Qualcomm Technologies, Inc. para crear un kit de desarrollo para visión de inteligencia artificial que corre bajo Azure IoT Edge, con ello tiene a su disposición el hardware y software necesario para desarrollar programas basados en 
cámara, con la que a su vez se pueden impulsar servicios de transmisión, cognitivos y aprendizaje automático. De forma general, se trabaja en realidad aumentada, big data, realidad virtual, tecnología de drones, inteligencia artificial, internet de las cosas (IoT), Intelligent Cloud y videojuegos [29].

Por otra parte, la empresa norteamericana S\&P Global Ratings, presentó un documento con las principales tendencias de la industria tecnológica para el año 2018, para ello se consultaron 4600 organizaciones debidamente seleccionadas por la empresa. En el reporte se menciona que el Software como Servicio (SaaS) es una tendencia, además que esto es muy bueno para las empresas porque es un bien tanto para clientes como para proveedores, algunas empresas que defienden SaaS son Intuit y Oracle. Se menciona que el uso del hardware ha disminuido debido a la migración a la nube, también que en 2018 disminuirán las ventas de semiconductores después de un buen año 2017 y la tendencia del software como servicio es una realidad. Por último, mencionan que existe una tendencia hacia tecnologías emergentes, entre ellas se habla sobre inteligencia artificial (aprendizaje automático y robótica), blockchain, realidad aumentada, realidad virtual y vehículos autónomos [3].

Silicon Valley se encuentra ubicada en California en los Estados Unidos y es conocido porque allí operan empresas informáticas de alta tecnología y el tema relevante en este momento es la cuarta revolución industrial, algunas de las empresas son: Facebook, Google, Apple, Tesla Motors, Netflix, Twitter, Airbnb, HP, SAP, LinkedIn, entre otras. Los temas tendencias son: desarrollo de software, inteligencia artificial y big data, robótica, realidad virtual y aumentada, economía compartida, internet de las cosas, tecnologías financieras, tecnologías móviles, computación en la nube y biotecnología [30], [31].

\section{Sector académico.}

Existen varios índices de medición para evidenciar la calidad académica de las instituciones de educación superior en el mundo, por ejemplo, los índices Shangai y QS. El índice QS cuenta con gran credibilidad tanto por su trayectoria como su objetividad. Entre los criterios de selección del Ranking se encuentran: la reputación académica, reputación de los empleadores, estudiantes de la facultad, docentes y estudiantes, citaciones por facultad, internacionalización de la facultad y estudiantes internacionales [32]. En la Tabla II se presenta el Ranking QS 2018 de las diez mejores universidades del mundo en ciencias de la computación y luego se presentan los temas que se trabajan en algunas de ellas.

En el MIT la tendencia son temas relacionados con biomedicina, biología computacional, computación exaescala, energía, IoT, robótica, tecnologías $5 \mathrm{~g}$, inteligencia artificial en la nube, machine learning, nanotecnología, construcción de software, Big data, creación de videojuegos, ciudades sensibles (ciudad con muchos sensores), impresión 3D, ciencia de datos. Entre muchos de los avances se menciona la creación de lenguajes de programación para computadores cuánticos, inteligencia artificial que permita recrear lugares a partir de imágenes, y seguridad para la tecnología Blockchain [33], [34].

TABLA II.

RANKING QS DE LAS MEJORES UNIVERSIDADES EN CIENCIAS DE LA COMPUTACIÓN EN EL MUNDO [32]

\begin{tabular}{|c|c|c|}
\hline Puesto & Universidad & País \\
\hline $\mathbf{1}$ & $\begin{array}{c}\text { Massachusetts Institute of Technology } \\
\text { (MIT) }\end{array}$ & United States \\
\hline $\mathbf{2}$ & Stanford University & United States \\
\hline $\mathbf{3}$ & Carnegie Mellon University & United States \\
\hline $\mathbf{4}$ & University of California Berkeley (UCB) & United States \\
\hline $\mathbf{5}$ & University of Cambridge & United Kingdom \\
\hline $\mathbf{6}$ & Harvard University & United States \\
\hline $\mathbf{7}$ & University of Oxford & United Kingdom \\
\hline $\mathbf{8}$ & Princeton University & United States \\
\hline $\mathbf{9}$ & ETH Zurich - Swiss Federal Institute of & Switzerland \\
\hline $\mathbf{1 0}$ & Technology & Singapure \\
\hline
\end{tabular}

Stanford University le interesa temas como inteligencia artificial incluyendo robótica, procesamiento de lenguaje natural, machine learning, biocomputación, ingeniería informática, simulación [2], algoritmos geométricos, interacción humano-computador, modelos de datos, lenguajes de consulta formales, extracción de información de fuentes de datos no estructurados, sistemas informáticos como compiladores, bases de datos, sistemas operativos, además teoría de la computación tales como estructuras de datos, algoritmos secuenciales y paralelos [35].

Carnegie Mellon University trabaja grandes áreas, entre ellas: ciencias de la computación, software, robótica, interacción humano computador, biología computacional, tecnologías del lenguaje y machine learning [36]. University of California Berkeley trabaja temas como exoesqueletos robóticos para discapacitados, partículas de "Smart Dust" para adquirir datos en tiempo real sobre nuestro entorno, para estos logros se incluyen en los currículos todos los temas como inteligencia artificial, interfaz humano-computador, big data, procesamiento de imágenes, analítica de datos, entre otros, los cuales permiten llegar a esos resultados [37].

En la Universidad de Cambridge se imparten cursos que tienen que ver con programación orientada a objetos con Java, sistemas operativos, diseño de computadores y redes, programación, algoritmos avanzados, construcción de compiladores, inteligencia artificial, seguridad, verificación de hardware y software, bioinformática, computación cuántica, interacción humano-computador, procesamiento de lenguaje natural, comercio electrónico, inteligencia artificial y gráficos avanzados [38].

La Universidad de Harvard menciona que las ciencias de la computación enseñan a pensar de forma más metódica y cómo resolver problemas de manera más efectiva, para ello cuenta con cursos relacionados con: software, teoría de la computación (algoritmos sobre neuronas, ADN y colonia de abejas), estructuras de datos, criptografía, machine learning, redes de computadores, compiladores, sistemas operativos, visualización, gráficos de computador, inteligencia artificial, sistemas autónomos de robots, software para computación científica, complejidad computacional, análisis probabilístico, 
algoritmos para big data, biología computacional, computación escalonada, seguridad de sistemas, sistemas de big data, visión por computador, machine learning para lenguaje natural, sistemas multiagente, interacción humanocomputador, comprensión de lenguaje natural [39].

Por otra parte, en la Tabla III se presentan los resultados del Ranking QS a nivel latinoamericano con las 10 mejores universidades en programas de ciencias de la computación y luego se mencionan los temas trabajados en algunas de ellas.

La Universidad de Chile tiene un programa en ciencias de la computación el cual tiene una duración de seis años, entre las temáticas tratadas se encuentran: programación, algoritmos y estructuras de datos, teoría de la computación, bases de datos, arquitectura de computadores, ingeniería de software, redes de computadores, evaluación de proyectos. La universidad investiga en ciencia e ingeniería de los datos (minería de datos, machine learning, computación en la nube, social analytics, crowdsourcing e inteligencia artificial), criptografía aplicada y seguridad computacional, interacción humano-computador, sistemas colaborativos, modelamiento geométrico, numérico y aplicaciones (algoritmos para triangulaciones, para mallas mixtas, tanto secuenciales como paralelos y aplicaciones en medicina, astronomía, computación gráfica y visualización científica, problemas en reconocimiento de imágenes) y representación del conocimiento [40].

TABLA III.

RANKING QS DE MEJORES UNIVERSIDADES EN CIENCIAS DE LA COMPUTACIÓN EN LATINOAMÉRICA [32].

\begin{tabular}{|c|c|c|}
\hline Puesto & Universidad & País \\
\hline $\mathbf{1}$ & Universidad de Chile & Chile \\
\hline $\mathbf{2}$ & $\begin{array}{c}\text { Universidad Nacional Autónoma de México } \\
\text { (UNAM) }\end{array}$ & México \\
\hline $\mathbf{3}$ & Universidade de Sao Paulo & Brasil \\
\hline $\mathbf{4}$ & Pontificia Universidad Católica de Chile (UC) & Chile \\
\hline $\mathbf{5}$ & $\begin{array}{c}\text { Instituto Tecnológico y de Estudios Superiores de } \\
\text { Monterrey }\end{array}$ & México \\
\hline $\mathbf{6}$ & Universidades Estadual de Campinas (Unicamp) & Brasil \\
\hline $\mathbf{7}$ & Instituto Politécnico Nacional (IPN) en México & México \\
\hline $\mathbf{8}$ & Universidad de Buenos Aires & Argentina \\
\hline $\mathbf{9}$ & Pontificia Universidade Católica do Rio de Janeiro & Brasil \\
\hline $\mathbf{1 0}$ & Universidad de los Andes & Colombia \\
\hline
\end{tabular}

En la Universidad Nacional Autónoma de México se cuenta con cursos sobre: estructuras discretas, gráficas y juegos, estructuras de datos, modelado y programación, autómatas y lenguajes formales, lógica computacional, computación distribuida, análisis de algoritmos, ingeniería de software, inteligencia artificial, redes de computadores, compiladores, computación concurrente, criptografía y seguridad, programación lineal, programación dinámica, arquitectura y diseño de software, computación cuántica, programación de videojuegos, visión por computador, lingüística computacional, robótica, entre otros [41].

La Universidad de Sao Paulo ofrece entre sus múltiples cursos: algoritmos y estructuras de datos, matemáticas discretas, sistemas digitales, programación orientada a objetos, algoritmos avanzados y aplicaciones, procesos estocásticos, ingeniería de software, programación concurrente, desarrollo web, ciencia de datos, inteligencia artificial, interacción humano-computador, redes neuronales, computación en la nube, arquitectura orientada a servicios, robótica, ingeniería en seguridad, web semántica, ingeniería de software para sistemas embebidos [42].

La Universidad de Buenos Aires es un referente académico a nivel suramericano entre otras cosas porque ha tenido un buen desempeño en maratones mundiales de programación, lo cual es un tema importante en la actualidad en ciencias computacionales. La institución cuenta con un programa en informática, el cual tiene una duración de 6 años, con tres ramas en la que el estudiante se puede especializar durante el curso de la carrera: i) gestión industrial, donde se prioriza el desarrollo de proyectos en entornos realistas; ii) sistemas distribuidos, donde se pretende una exigente formación técnica; y iii) sistemas de producción, donde se pretende que el estudiante pueda automatizar procesos industriales complejos. Para lograrlo se estudian cursos del componente matemático, también administrativo y de proyectos, además asignaturas como algoritmos y programación, modelos de optimización, técnicas de diseño, bases de datos, circuitos, programación concurrente, sistemas distribuidos, sistemas automáticos de diagnóstico y detección de fallas, teoría de control, robótica, procesamiento del habla e imágenes, sistemas biológicos, simulación, criptografía y seguridad, lenguajes formales, arquitectura de software, entre otros [43].

Por otra parte, desde el año de 2008 la Association for Computer Machinery (ACM) y la Computer Society of the Institute for Electrical and Electronics Engineers (IEEE) decidieron unirse para desarrollar una serie de documentos que se puedan utilizar como guía curricular para los programas en computación. En el año 2017 el documento se denomina "Computing Curricula Guidelines for Undergraduate Degree Programs in Information Technology 2017" o se puede utilizar la sigla IT2017. En éste se presentan unos temas generales de tendencia, en los cuales se basan para la creación del currículo, los temas son: aplicaciones móviles, plataformas sociales, desarrollo de software, experiencias de usuario, internet de las cosas y big data en los que se incluyen temas como analítica de datos y computación en la nube, ciberseguridad, sistemas web, escalabilidad y análisis de datos, sistemas virtuales y servicios, redes aplicadas, robótica, ingeniería de software, finalmente automatización, donde se intenta automatizar las capacidades cognitivas [5]. Se menciona en el documento que son necesarias tres dimensiones para el profesional en TI: i) el conocimiento; ii) las habilidades (dentro de la profesión); y iii) la disposición (relaciones interpersonales).

\section{Desafios actuales de la disciplina.}

Ovalles Pabón [44] menciona que los retos para los profesionales en TIC se definen de acuerdo al contexto, también a los desafíos que la globalización de la economía le imponga. Algunos de los retos son la actualización permanente, resolución de problemas, creatividad e innovación, dominio de una segunda lengua, uso y desarrollo de las TIC, creación de empresas de base tecnológica y cuidado del medio ambiente. La Agencia Nacional de 
Ingeniería de los Estados Unidos (ANE) planteó 14 desafíos para la ingeniería, tres de ellos relacionados con las TIC: i) avanzar en la informática para la salud; ii) proteger el ciberespacio; y iii) enriquecer la realidad virtual.

Henao et al. [45] menciona que se puede crear una estrategia de colaboración Universidad-Industria, mediante la creación en las Universidades de líneas de formación las cuales estén más cercanas a las líneas de negocio de las empresas de desarrollo y que esas líneas de formación estén relacionadas con las líneas de producción que maneja la industria de software. Para realizar este despliegue, se propone el reto que los estudiantes de ciencias de la computación realicen proyectos de innovación y desarrollo, en temas propuestos por empresas del sector de desarrollo de software, los trabajos deben ser dirigidos por el ingeniero de la empresa y el docente tutor de la investigación. Es un gran desafío, pero se podría tener en cuenta para mejorar la calidad del software y sobre todo son pertinentes para ir formando al estudiante en la academia con problemas de la vida real que se dan en las empresas.

Uno de los grandes desafíos para los programas de ciencias de la computación, es aumentar el número de estudiantes y aunque es una problemática que se presenta a nivel mundial, en cada país es necesario capacitar nuevos profesionales dada la alta demanda existente en la actualidad [46].

Otro de los desafíos, es enseñar ciencias básicas para futuros ingenieros [47]. Los autores presentan una serie de reflexiones teóricas, a partir de trabajos publicados previamente, en el que se trata de resaltar la importancia de trabajar en esa dirección, dado a que cada día existen menos ingenieros y se requieren muchos más, es decir, se requiere satisfacer las necesidades inmediatas de la industria. Cabe resaltar que muchos estudiantes temen a la ingeniería, por las asignaturas de ciencias básicas y por lo tanto es un desafío importante [48]-[50].

Un reto importante para las personas que trabajan en ciencias de la computación, es el conocimiento del idioma inglés, debido a que los lenguajes de programación, la documentación en computación, los procesos investigativos a nivel de posgrado, la gestión de proyectos a nivel internacional, el soporte técnico a clientes extranjeros, las certificaciones internacionales, entre otros, requieren que los estudiantes hayan desarrollado competencias muy altas en este idioma durante sus carreras. Es necesario que las universidades apliquen estrategias efectivas, para que los futuros profesionales de los campos de la computación cuenten con un buen nivel de inglés para el desempeño de su profesión [51].

\section{RESULTADOS Y DISCUSIÓN}

Los resultados muestran que existen muchos temas importantes en ciencias de la computación, los cuales son actuales y de interés para muchos sectores, porque contribuyen en gran medida al desarrollo de los países. En la Tabla IV se sintetizan los temas que abordan los gobiernos líderes en tecnología, la cual aplican para generar desarrollo económico y cultural.
Los países mencionados en la Tabla IV, utilizan la tecnología para beneficio de la sociedad, hacen grandes apuestas por una interconexión utilizando Internet banda ancha para beneficio de las personas, también propenden porque dispongan de dispositivos inteligentes para acceder a esa red. En países como Estados Unidos, Inglaterra y Alemania utilizan la tecnología de Big Data para el crecimiento económico. Japón, es un país que hace muchos años trabaja en robótica y vehículos autónomos. Otros países de Europa trabajan en la gestión de la información para beneficio de los estados. Finalmente, en muchos países, el desarrollo de software es parte fundamental a la hora de mejorar procesos, pero actualmente no sólo basta con la creación de aplicaciones, el software debe ir más allá y por eso actualmente se está aplicando el concepto de Inteligencia Artificial, para tratar de desarrollar aplicaciones más avanzadas y que se puedan utilizar para el desarrollo económico de los países.

Por otra parte, entre muchas temáticas que se manejan en el sector empresarial en computación, en la Tabla $\mathrm{V}$ se presentan los temas recurrentes que abordan las empresas líderes en tecnología.

Las empresas mostradas en la Tabla $\mathrm{V}$, también se encuentran en las primeras posiciones de muchos de las clasificaciones de las mejores empresas a nivel mundial, es decir, la tecnología actualmente es un negocio rentable y que las empresas productivas sienten la necesidad de identificar aquellas temáticas con las que pueden mejorar su productividad. Por ejemplo, Amazon, empezó como una empresa de comercio electrónico, luego identificó otras oportunidades de negocio y actualmente investiga en tecnología para aplicarla y seguir creciendo a tal nivel que se ha convertido en una de las mejores compañías. Los resultados también muestran que las empresas establecidas en Silicon Valley, marcan la pauta en temas de tecnología.

Finalmente, los temas representativos que se abordan en el sector académico, se presentan en la Tabla VI, así como los temas de interés para las principales universidades a nivel latinoamericano.

Según los resultados, es claro que para todo proceso de desarrollo tecnológico es indispensable el software, con este elemento se pueden abordar otras tecnologías que van a la par de éste. Por ejemplo, con la Inteligencia Artificial se trata de darle un poco de inteligencia dispositivos electrónicos para que realicen tareas aún más especializadas de manera autónoma [52]. La tecnología de Big Data, consiste en agrupar grandes cantidades de información de distintos tipos, para luego procesarla a través de software especializado. Así podría pasar con tecnologías como realidad virtual y aumentada, sistemas autónomos, videojuegos, biomedicina, etc. Para optimizar el uso de cualquier sistema mencionado, se requiere que se manejen aspectos importantes como el software, además la seguridad de esos sistemas debido a que son las bases para la nueva era de la computación, la cual lleva consigo temas como la computación cuántica, la cual requerirá tanto nuevo hardware como software. 
TABLA IV.

SÍNTESIS DE TEMAS IMPORTANTES EN EL SECTOR GUBERNAMENTAL.

\begin{tabular}{|c|c|c|c|c|c|c|c|c|c|c|c|c|c|}
\hline \multicolumn{14}{|c|}{ SÍNTESIS DE TEMAS IMPORTANTES EN EL SECTOR GUBERNAMENTAL. } \\
\hline País & 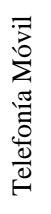 & 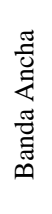 & 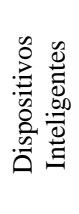 & $\begin{array}{l}\stackrel{\Xi}{0} \\
0 \\
.0 \\
0\end{array}$ & 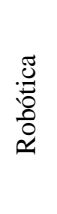 & 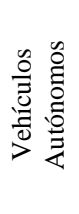 & 官 & $\begin{array}{l}0 \\
0 \\
0 \\
0 \\
0 \\
0 \\
0 \\
0 \\
0\end{array}$ & 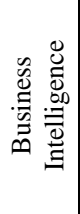 & 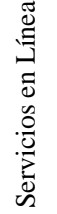 & 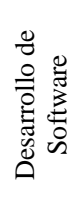 & 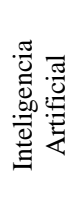 & 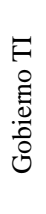 \\
\hline Singapur & $\mathrm{X}$ & $\mathrm{X}$ & $\mathrm{X}$ & & & & & & & & $\mathrm{X}$ & $X$ & $\mathrm{X}$ \\
\hline Finlandia & & $\mathrm{X}$ & $\mathrm{X}$ & & & & & & & & $\mathrm{X}$ & & $\mathrm{X}$ \\
\hline Suecia & & $\mathrm{X}$ & $X$ & & & & & & & & $X$ & & $\mathrm{X}$ \\
\hline Noruega & & $\mathrm{X}$ & & & & & & & & & $\mathrm{X}$ & $X$ & $\mathrm{X}$ \\
\hline U.S.A & & $X$ & $\mathrm{X}$ & $\mathrm{X}$ & & & & & & & $\mathrm{X}$ & $\mathrm{X}$ & $\mathrm{X}$ \\
\hline Inglaterra & & & & $\mathrm{X}$ & & & & & & & $\mathrm{X}$ & $\mathrm{X}$ & $\mathrm{X}$ \\
\hline Japón & & & & & $\mathrm{X}$ & $\mathrm{X}$ & & & & & $\mathrm{X}$ & $\mathrm{X}$ & $\mathrm{X}$ \\
\hline Holanda & & & & & & & $X$ & & & & $\mathrm{X}$ & & $\mathrm{X}$ \\
\hline Suiza & & & & & & & & $X$ & & & $\mathrm{X}$ & & $\mathrm{X}$ \\
\hline Alemania & & & & $X$ & & & & & $\mathrm{X}$ & & $\mathrm{X}$ & $\mathrm{X}$ & $\mathrm{X}$ \\
\hline Francia & & & & & & & & & & $\mathrm{X}$ & $\mathrm{X}$ & & $\mathrm{X}$ \\
\hline
\end{tabular}

TABLA V

SÍNTESIS DE TEMAS IMPORTANTES EN EL SECTOR EMPRESARIAL.

\begin{tabular}{|c|c|c|c|c|c|c|c|c|c|c|c|c|}
\hline Empresa & 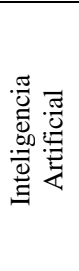 & 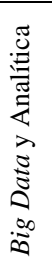 & 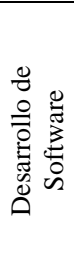 & 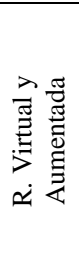 & 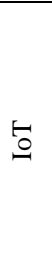 & 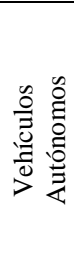 & 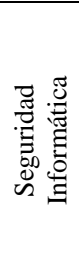 & 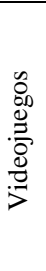 & 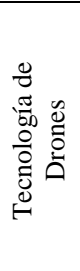 & 惢 & 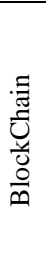 & 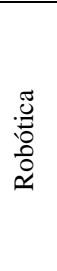 \\
\hline Amazon & $\mathrm{X}$ & $\mathrm{X}$ & $X$ & & & & & & & & & \\
\hline Apple & $\mathrm{X}$ & & $\mathrm{X}$ & $\mathrm{X}$ & & & & & & & & \\
\hline Google & $\bar{X}$ & $\mathrm{X}$ & $\mathrm{X}$ & $\mathrm{X}$ & & $\mathrm{X}$ & $\bar{X}$ & & & $\bar{X}$ & & \\
\hline Samsung & $\mathrm{X}$ & & $\mathrm{X}$ & & $\mathrm{X}$ & & & & & & & \\
\hline Facebook & $\mathrm{X}$ & $\mathrm{X}$ & $\mathrm{X}$ & $\mathrm{X}$ & & & $\mathrm{X}$ & & & $\mathrm{X}$ & & \\
\hline Microsoft & $X$ & $\mathrm{X}$ & $\mathrm{X}$ & $\mathrm{X}$ & $\mathrm{X}$ & & $\mathrm{X}$ & $\mathrm{X}$ & $\mathrm{X}$ & & & \\
\hline Silicon Valley & $\mathrm{X}$ & $\mathrm{X}$ & $\mathrm{X}$ & $\mathrm{X}$ & $\mathrm{X}$ & $\mathrm{X}$ & $\mathrm{X}$ & $\mathrm{X}$ & $\mathrm{X}$ & $\mathrm{X}$ & $\mathrm{X}$ & $\mathrm{X}$ \\
\hline
\end{tabular}

TABLA VI.

SíNTESIS DE TEMAS IMPORTANTES EN EL SECTOR ACADÉMICO.

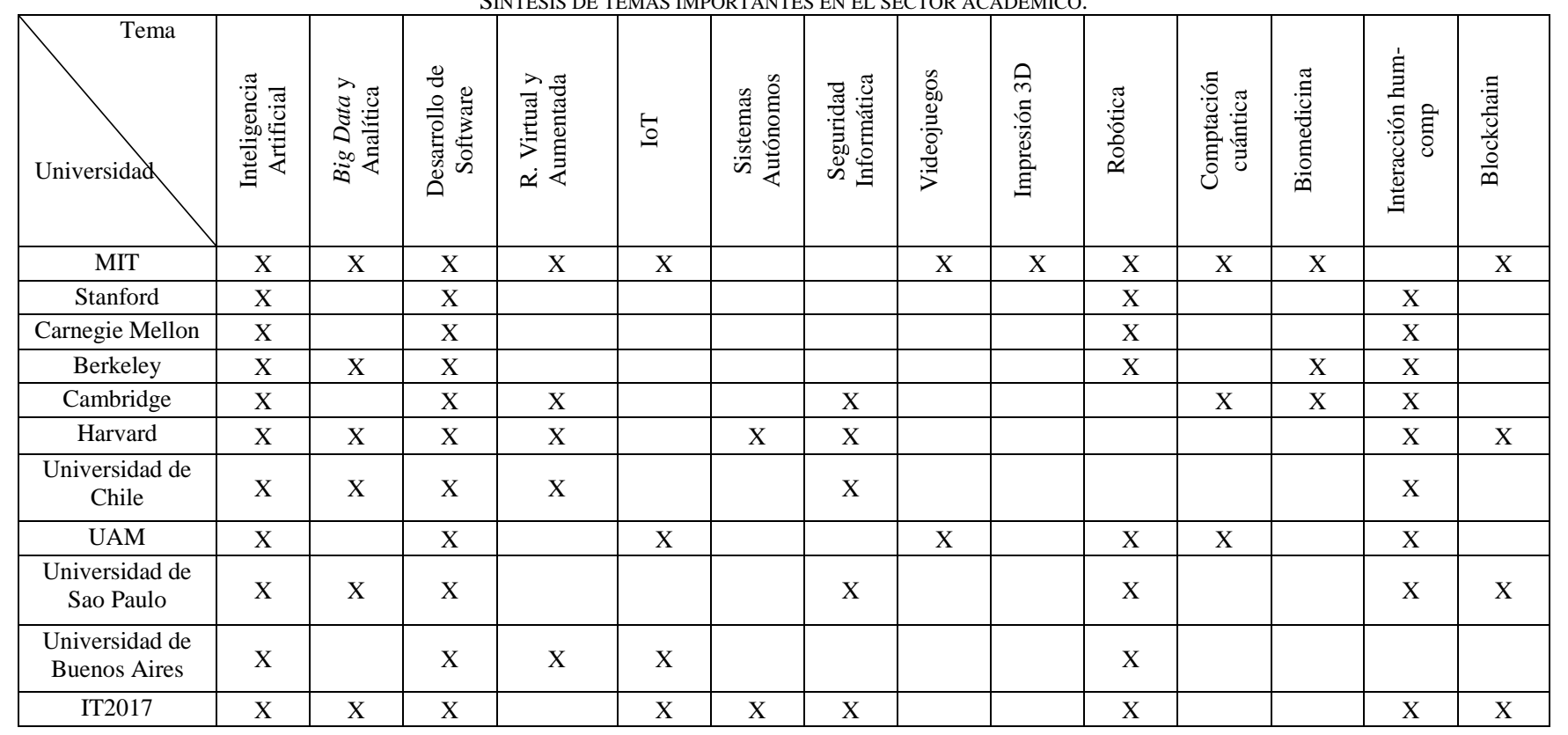




\section{CONCLUSIONES}

En este estudio de tendencias se realizó una revisión bibliográfica en ciencias de la computación a nivel internacional, ello teniendo en cuenta los entes gubernamentales, las empresas y la academia. También se plasmaron algunos desafíos de la profesión. Con ello se pretende realizar un aporte a la disciplina.

Se encontró que los principales gobiernos del mundo, quieren mejorar los servicios que ofrecen a través de la optimización de procesos con el uso de las tecnologías de información. Los gobiernos quieren implementar sistemas con un grado de inteligencia, los cuales permitan agilizar procesos importantes. Para lograr ese objetivo es necesario llevar a cabo proyectos ambiciosos en TI, de esa forma tratar de mejorar la calidad de vida de las personas, lo cual se convierte en un aporte hacia el desarrollo de los países.

Además de lo anterior, las universidades tienen una gran responsabilidad, debido a que es allí donde se realiza la experimentación de nuevas tecnologías. Las principales universidades en ciencias de la computación a nivel mundial según el Ranking QS trabajan temas como: biomedicina, IoT, robótica, inteligencia artificial, construcción de software, big data, creación de videojuegos, impresión 3d, ciencia de datos, lenguajes de programación para computadores cuánticos, blockchain, interacción humano-computador, computación cuántica, entre muchas más.

Adicionalmente, se puede concluir que algunos desafíos para los programas de ciencias de la computación pueden ser: i) buscar que los estados inviertan en ciencia y tecnología; ii) avanzar en la informática para la salud, proteger el ciberespacio y enriquecer la realidad virtual; iii) lograr que los estudiantes de ciencias de la computación realicen proyectos de innovación y desarrollo en temas propuestos por empresas, también que puedan integrar la teoría con la práctica; iv) aumentar el número de estudiantes a nivel mundial; v) formar egresados de alta calidad y gran capacidad de evolución; vi) mejorar la enseñanza de las ciencias básicas en ingeniería; vii) fortalecer las competencias genéricas (trabajo en equipo, razonamiento crítico, capacidad de investigación, entre otras.), así como viii) incentivar el aprendizaje de una segunda lengua, preferiblemente inglés.

\section{REFERENCIAS}

[1] M. Sachon, «Cuando personas y máquinas trabajan juntos: Los pilares de la industria 4.0», IEEM Rev. Negocios, n. ${ }^{\circ}$ 2, pp. 46-54, abr. 2018.

[2] E. Salimi, A. H. Cadenbach, y A. E. Abbas, «Survey of Four Uncertainty Quantifications Methods in Systems Engineering», en Disciplinary Convergence in Systems Engineering Research, Springer, Cham, 2018, pp. 945-957.

[3] A. Chang, D. Tsui, R. Hsu, y T. Guermann, «Industry Top Trends 2018: Technology», S\&P Global Ratings, pp. 181-190, 2018.

[4] Academia Colombiana de Ciencias Exactas, Físicas y Naturales, «Desafíos para el 2030: Ciencia, tecnología, educación y medio ambiente». Maloka -MinEducación, 2018.

[5] IT2017 Task Group, Information Technology Curricula 2017: Curriculum Guidelines for Baccalaureate Degree Programs in Information Technology. USA: Association for Computing Machinery ACM \& IEEE, 2017.
[6] G.-J. Correa-Henao, J.-M. Gaviria-Hincapié, R. A. Giraldo-Escobar, G.-J. Correa-Henao, J.-M. Gaviria-Hincapié, y R. A. Giraldo-Escobar, «Revisión y diagnóstico sobre las condiciones de calidad en los programas de Ingeniería de Sistemas en Colombia», Rev. Iberoam. Educ. Super., vol. 8, n. ${ }^{\circ} 22$, pp. 3-24, 2017.

[7] S. J. Martínez Marín, S. Arango Aramburo, y J. Robledo Velásquez, «El crecimiento de la industria del software en colombia: un análisis sistémico», Rev. EIA, vol. 12, n. ${ }^{\circ} 23$, pp. 95-106, 2015.

[8] C. S. Montes y C. S. Montes, «Graduate and Job Profile in the Context of Technological Advancement», Pedagog. Saberes, n. ${ }^{\circ} 48$, pp. 83-96, jun. 2018.

[9] BID, «Servicios sociales para ciudadanos inteligentes digitales: Oportunidades para América Latina y el Caribe. » Banco Interamericano de Desarrollo, 2017.

[10] L. Bassi, «Industry 4.0: Hope, hype or revolution?», en 2017 IEEE 3rd International Forum on Research and Technologies for Society and Industry (RTSI), Modena, Italy, 2017, pp. 1-6, doi: 10.1109/RTSI.2017.8065927.

[11] T. M. Mitchell, J. Betteridge, A. Carlson, E. Hruschka, y R. Wang, «Populating the semantic web by macro-reading internet text», en International Semantic Web Conference, vol. 5823, Berlín: Springer, 2009, pp. 998-1002.

[12] B. Chakravorti y R. Chaturvedi, Digital Planet 2017: How competitiveness and trust in digital economies vary across the world. Somerville, USA: The Fletcher School, Tufts University, 2017.

[13] S. Baller, S. Dutta, y B. Lanvin, The Global Information Technology Report: Innovating in the Digital Economy. Geneve: World Economic Forum, 2016.

[14] G. P. M. Góngora y W. N. Bernal, «Gestión de tecnología de información para gobiernos inteligentes: un enfoque de arquitectura empresarial», Rev. Espac., vol. 38, n. ${ }^{\circ}$ 42, pp. 14-24, 2017.

[15] Forbes, «Portal Forbes», Forbes, 2018. [En línea]. Disponible en: https://www.forbes.com/. [Accedido: 11-abr-2018].

[16] B. Finance, «Brand Finance Global 500 2018: The annual report on the world's most valuable brands», Brand Finance, London, 2018.

[17] Amazon, «Amazon.com: Online Shopping for Electronics, Apparel, Computers, Books, DVDs \& more», Amazon, 2018. [En línea]. Disponible en: https://www.amazon.com/. [Accedido: 03-jul-2018].

[18] EFE, «Amazon abre este lunes su primer supermercado sin cajeros», Portafolio.co, 2018. [En línea]. Disponible en: http://www.portafolio.co/negocios/amazon-abre-este-lunes-su-primersupermercado-sin-cajeros-513464. [Accedido: 03-jul-2018].

[19] A. García, «Ciudades inteligentes: un mercado de 1,29 billones de euros - elEconomista.es», El Economista, 2018. [En línea]. Disponible en: $\quad$ http://www.eleconomista.es/empresasfinanzas/noticias/8581426/09/17/Ciudades-inteligentes-un-mercadode-129-billones-de-euros.html. [Accedido: 04-jul-2018].

[20] A. Estévez Martín y B. Ramírez Barredo, «SMARTCITY: La inteligencia artificial en la ciudad del futuro: Estudio del caso Amazon Go. », en VI Congreso Internacional Ciudades Creativas, Orlando, Florida, USA, 2018, pp. 199-215.

[21] Á. Jiménez De Luis, «WWDC 2018: Apple pone toda la carne en el asador con iOS 12 y MacOS Mojave», EL MUNDO: Tecnología, 04jun-2018. [En línea]. Disponible en: http://www.elmundo.es/tecnologia/2018/06/04/5b157cea22601dd15b8 b45f7.html. [Accedido: 06-jul-2018].

[22] I. Bădărînză, A. Sterca, y F. M. Boian, «Using the User's Recent Browsing History for Personalized Query Suggestions», en 2018 26th International Conference on Software, Telecommunications and Computer Networks (SoftCOM), Split, Croatia, 2018, pp. 1-6, doi: 10.23919/SOFTCOM.2018.8555774.

[23] G. Sama, «Google I/O 2018: Lo más importante de la conferencia anual de Google», CNET en Español, 2018. [En línea]. Disponible en: https://www.cnet.com/es/noticias/google-io-2018-la-semana-cnet/. [Accedido: 06-jul-2018].

[24] S. Tibken, «Bixby, televisor The Wall y AI: Todo lo que Samsung presentó en CES 2018», CNET en Español, 2018. [En línea]. Disponible en: https://www.cnet.com/es/noticias/smartthings-bixby-ytvs-todo-lo-que-samsung-presento-en-ces-2018/. [Accedido: 06-jul2018].

[25] I. Luzardo, «Esto mostrará Samsung en el CES 2018», ENTER.CO, 2018. [En línea]. Disponible en http://www.enter.co/eventos/ces/2018/samsung-en-el-ces/. [Accedido: 06-jul-2018]. 
[26] Facebook, «Facebook para desarrolladores: Conferencia F8», Facebook para desarrolladores, 2018. [En línea]. Disponible en: https://www.f8.com/. [Accedido: 09-jul-2018].

[27] K. Hazelwood et al., «Applied Machine Learning at Facebook: A Datacenter Infrastructure Perspective», en 2018 IEEE International Symposium on High Performance Computer Architecture (HPCA), Vienna, Austria, 2018, pp. 620-629, doi: 10.1109/HPCA.2018.00059.

[28] J. Monfort, «El E3 2018 de Microsoft: Todas las novedades y anuncios», Hipertextual: Videojuegos, 2018. [En línea]. Disponible en: https://hipertextual.com/2018/06/microsoft-e3-2018-anunciosresumen. [Accedido: 09-jul-2018].

[29] Microsoft, «Microsoft Build 2018», Microsoft Build 2018 Developer Conference, 2018. [En línea]. Disponible en: https://microsoft.com/enus/build. [Accedido: 09-jul-2018].

[30] Silicon Valley, «Silicon Valley Innovation Center», Silicon Valley Innovation Center, 2018. [En línea]. Disponible en: https://siliconvalley.center/. [Accedido: 10-jul-2018].

[31] T. Wang, L. Wang, y T. Chen, «Secrets of success: Innovation in the Silicon Valley: Business development and innovation of tech companies», en 2014 International Conference on Behavioral, Economic, and Socio-Cultural Computing (BESC2014), Shanghai, China, 2014, pp. 1-5, doi: 10.1109/BESC.2014.7059530.

[32] QS, «QS World University Rankings 2019», QS World Top Universities, 2019. [En línea]. Disponible en: https://www.topuniversities.com/university-rankings/worlduniversity-rankings/2018. [Accedido: 14-jul-2018].

[33] MIT, «The Massachusetts Institute of Technology (MIT)», MIT, 2018. [En línea]. Disponible en: http://web.mit.edu/. [Accedido: 15-jul2018].

[34] A. D. Silva et al., «Fuzzy Modeling for Predicting Patient Survival Rate in ICU with AKI», en 2018 IEEE International Conference on Fuzzy Systems (FUZZ-IEEE), Rio de Janeiro, Brazil, 2018, pp. 1-6, doi: 10.1109/FUZZ-IEEE.2018.8491678.

[35] Stanford, «Stanford University», Stanford University, 2018. [En línea]. Disponible en: https://www.stanford.edu/. [Accedido: 16-jul2018].

[36] Carnegie Mellon, «Carnegie Mellon University - CMU», Homepage CMU Carnegie Mellon University, 2018. [En línea]. Disponible en: http://www.cmu.edu/index.html. [Accedido: 16-jul-2018].

[37] Berkeley University, «Home University of California, Berkeley», University of California, Berkeley, 2018. [En línea]. Disponible en: https://www.berkeley.edu/. [Accedido: 16-jul-2018].

[38] University of Cambridge, «University of Cambridge - Computer Science Program», Undergraduate Study - Computer Science, 2018. [En línea]. Disponible https://www.undergraduate.study.cam.ac.uk/courses/computerscience. [Accedido: 16-jul-2018].

[39] University of Harvard, «Harvard University Home», Harvard University, 2018. [En línea]. Disponible en: http://www.harvard.edu/. [Accedido: 16-jul-2018].

[40] Universidad de Chile, «Universidad de Chile - Homepage», Universidad de Chile, 2018. [En línea]. Disponible en: http://www.uchile.cl. [Accedido: 16-jul-2018].

[41] UNAM, «Portal UNAM», Universidad Nacional Autónoma de México, 2018. [En línea]. Disponible en: https://www.unam.mx/. [Accedido: 16-jul-2018].

[42] Universidade de Sao Paulo, «USP - Universidade de São Paulo Home», Universidade de São Paulo, 2018. [En línea]. Disponible en: http://www5.usp.br/. [Accedido: 16-jul-2018].

[43] UBA, «Universidad de Buenos Aires - Homepage», Universidad de Buenos Aires, 2018. [En línea]. Disponible en: http://www.uba.ar/. [Accedido: 16-jul-2018].

[44] F. O. Ovalles Pabón, «Retos y tendencias de la Ingeniería en Tecnologías de la Información y las Comunicaciones (TIC) frente al Desarrollo del Sector Productivo», Rev. Espac., vol. 39, n. ${ }^{\circ}$ 14, pp. 7$19,2018$.

[45] C. F. Henao Villa, D. A. García Arango, E. D. Aguirre Mesa, y G. A. Araque González, «Implementación de industria de software en programas de ingeniería de sistemas: Descripción del proceso para la Corporación Universitaria Americana», 2017.

[46] Ernesto Amaru Galvis-Lista, Mayda Patricia González-Zabala, y Jenny Marcela Sánchez-Torres, «Estudio exploratorio sobre factores de éxito y barreras para la gestión de conocimiento en organizaciones desarrolladoras de software en Colombia», CLIO América Vol 11 Iss
21 2017, vol. 11, n. ${ }^{\circ}$ 21, pp. 48-58, 2017, doi: $10.21676 / 23897848.2114$.

[47] R. Rodríguez, «Repensando la enseñanza de las matemáticas para futuros ingenieros: actualidades y desafíos», IE Rev. Investig. Educ. REDIECH, vol. 8, n. ${ }^{\circ} 15$, pp. 69-85, 2017.

[48] E. Ramírez Sinarahua, S. Concalvez, y C. Augusto, «Aplicación del software Matlab como instrumento de enseñanza de matemática en estudiantes del I ciclo de Ingeniería de Sistemas de la Universidad Nacional de la Amazonía Peruana 2017», Tesis de Pregrado, Universidad Nacional de la Amazonía Peruana, Perú, 2017.

[49] R. A. Bustos González, «Tutoría entre pares como estrategia para la formación de ingenieros: desafíos y nudos críticos», Rev. Educ. En Ing., vol. 13, n. ${ }^{\circ}$ 26, pp. 4-10, jul. 2018, doi: 10.26507/rei.v13n26.887.

[50] E. M. F. Ramírez, A. S. Castillo, I. A. Berenguer, y A. G. S. A. G. Sánchez, «Consideraciones epistemológicas sobre la formación investigativa del licenciado en ciencia de la computación», Rev. Órbita Pedagógica, vol. 2, n. ${ }^{\circ}$ 2, pp. 45-68, mar. 2018.

[51] R. F. M. Pacheco y Y. T. Fernández, «Lógica didáctico-tecnológica del inglés con fines profesionales para el perfil de la ingeniería informática», Rev. Electrónica Diálogos Educ., vol. 11, n. ${ }^{\circ} 22$, pp. 6698, jul. 2017.

[52] D. Ostrowski, «Artificial Intelligence with Big Data», en 2018 First International Conference on Artificial Intelligence for Industries (AI4I), Laguna Hills, CA, USA, 2018, pp. 125-126, doi: 10.1109/AI4I.2018.8665678.

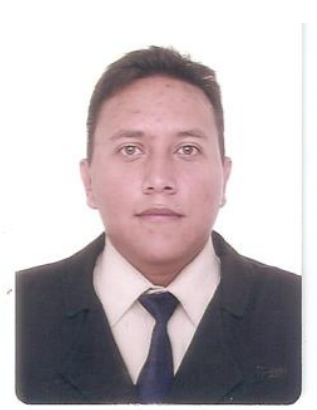

Juan Carlos Blandón Andrade. Ingeniero de Sistemas de la Universidad Cooperativa de Colombia 2004. Magister en Ingeniería énfasis en sistemas y computación de la Universidad Javeriana sedede Cali Colombia 2011. Ph.D. en Ingeniería - Sistemas e Informática de la Universidad Nacional de Colombia sede Medellín 2017. Actualmente es professor Asociado de la Unversidad Católica de Pereira, Colombia. Sus intereses de investigación están relacionados con Ingeniería de Software e Inteligencia Artificial en el área de Procesamiento de

Lenguaje Natural (PLN)

ORCID: http://orcid.org/0000-0003-1566-1832 